ABS11: Updating knowledge of primary care physicians on the management of asthma using a new interactive method based on Playing Cards

Louis-Philippe Boulet ${ }^{a}$, Francine Borduas ${ }^{a}$, Jacques Bouchard ${ }^{a}$, Johanne Blais $^{\mathrm{a}}$, Frederick E. Hargreave ${ }^{\mathrm{b}}$, Michel Rouleau ${ }^{\mathrm{c}}$

a Laval Hospital and University, 2725 Chemin Sainte-Foy, Quebec City, Canada G1V 4 G5 ${ }^{\text {b } M C M a s t e r ~ U n i v e r s i t y, ~ C a n a d a ~}{ }^{\mathrm{C}}$ Laval University, Canada

Introduction: Asthma control and care are still frequently sub-optimal and new means of improving knowledge transfer to caregivers, such as those based on Game-based methods, are needed [1]. Aims and objectives: To describe an innovative method of updating Primary Care Physicians (PCP) on asthma management, using a method integrating Play and Scientific components. Methods: We developed an interactive Playing Card workshop to communicate Asthma Guidelines recommendations and facilitate knowledge transfer. During this workshop, the physicians had to manage real-life cases using the tools that were provided to them and discuss with their peers and asthma specialists about the optimal management of the cases proposed. Results: The game allowed the physician to communicate relevant content, experiment with a different learning format and stimulated interactivity in a climate of friendly competition. An initial assessment made by thirty-four participating physicians showed that the method was considered innovative, highly interactive and useful in promoting reflection, interaction and learning. It generated relevant discussions on how to apply Guidelines recommendations into current asthma care. Conclusions: This new interactive educational intervention, integrating play and scientific components, was considered an innovative and valuable method by participants. It stimulated reflection and generated relevant discussion on current asthma Guidelines and how to apply them to current practice, thus facilitating knowledge transfer to caregivers

Conflict of interest and funding

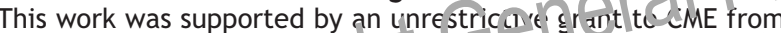
Merck Frosst Canada Ltd

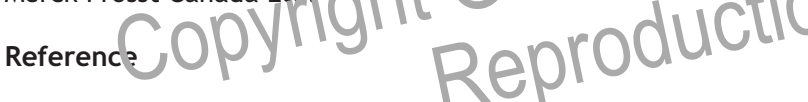

[1] Foreman J. NEXT-Generation. Educational Technology versus the Lecture. Educause Rev 2003;38:13-22.

doi:10.1016/j.pcrj.2006.04.113

\section{ABS12: Patient views of the patient asthma APGAR}

Barbara P. Yawn, Susan Bertram, Peter Wollan

Olmsted Medical Center, 210 Ninth St SE, Rochester Minnesota, 55904, USA

Background: Collecting health information directly from patients prior to a physician visit can save physician time and may provide patients additional ability to guide asthma visits. Objective: To assess patients (and parents) opinions regarding the use of the Patient ASTHMA APGAR during asthma related physician visits. Sample: Patients and parents of patients $(n=83)$ from seven rural family medicine practices who participated in the pilot study of the Patient ASTHMA APGAR. Methods: On-site focus groups in the seven sites with immersion-crystallization analysis. Sites were spread from Pennsylvania to Washington state with 4 sites within Minnesota. Results: The 83 participants included 12 children and adolescents, 10 parents of children or adolescents with asthma and 61 adults with asthma. The age ranged from 6 years to 78 years and 17 of the attendees were men or boys. The group reported no dislikes or problems with the form, found it to be easily readable and could be completed in five to seven minutes. The groups' impression of the value of the ASTHMA APGAR fell into six major domains: improved focus of asthma visits, identification of triggers, better communication with physicians, specific needs-focused education provided, better asthma care and ability to assess control asthma at home. Specific quotations will be provided to illustrate each domain. Every attendee desired to have their physician's office continue using the form. Conclusion: These volunteer focus group members found the completion of a simple asthma assessment form to be useful in the conduct and outcomes of visits for routine asthma care as well as visits for asthma attacks.

\section{Conflict of interest and funding}

No conflicts of interest and funding was provided by the Agency for HealthCare Research and Quality.

doi:10.1016/j.pcrj.2006.04.114

ABS13: Systematic practice-based asthma care in the Australian setting

Justin Beilby ${ }^{a}$, Christine Holton $^{a}$, Mark Harris ${ }^{b}$, Judy Proudfoot $^{b}$, Clare Harper ${ }^{b}$, Emmae Ramsay ${ }^{a}$, Richard Ruffin ${ }^{a}$

a The University of Adelaide, North Terrace, Adelaide, 5005, Australia ${ }^{b}$ University of New South Wales, Australia

Introduction: Asthma is a major health priority in Australia. Many patients are not being adequately managed in primary care, despite the introduction of incentives encouraging a more comprehensive and systematic approach to care. Aims and objectives: To evaluate whether systematic asthma care (especially the use of recall for an asthma review) improves the quality of care and health outcomes for adult patients, and whether this is cost effectiv. Subject and method: We recruited 565 patients ased 10 or c vel who had been diagnosed with incderalte or severe asthma. They were from 40 general prac i.e; in uban and rural settings in two Australian st at $E$ S. IV conducted a cluster randomised controlled trial, with the practice as the ani of randomisation. The intervention included

sotting up an asthma register-recall system incorporating postcard prompts;

- education of GPs and staff about their roles in systematic care, use of spirometry and the Asthma 3+ Plan.

Data was collected through patient questionnaires, spirometry, case note review, and interviews with practice staff. Results: Preliminary analysis revealed that $47 \%$ of patients responded to the postcard prompts and made appointments for an asthma review. Final results will be presented showing whether this resulted in any significant differences between intervention and control patients in

- quality of care - including use of spirometry, asthma action plans, education;

- clinical outcomes - including lung function, asthma on waking, days off work;

- acceptability of systematic care;

- quality of life.

Conclusions: The trial will determine whether changes in delivery of asthma care are acceptable, feasible and effective in improving patient outcomes - and provide an evidence base for future policy initiatives.

Conflict of interest and funding

No conflicts of interest. Funded by National Health and Medical Research Council of Australia.

doi:10.1016/j.pcrj.2006.04.115 